\title{
A!
}

This is an electronic reprint of the original article.

This reprint may differ from the original in pagination and typographic detail.

Kaladzhyan, Vardan; Röntynen, Joel; Simon, Pascal; Ojanen, Teemu

\section{Topological state engineering by potential impurities on chiral superconductors}

Published in:

Physical Review B

DOI:

10.1103/PhysRevB.94.060505

Published: 11/08/2016

Document Version

Publisher's PDF, also known as Version of record

Please cite the original version:

Kaladzhyan, V., Röntynen, J., Simon, P., \& Ojanen, T. (2016). Topological state engineering by potential impurities on chiral superconductors. Physical Review B, 94(6), 1-5. [060505].

https://doi.org/10.1103/PhysRevB.94.060505

This material is protected by copyright and other intellectual property rights, and duplication or sale of all or part of any of the repository collections is not permitted, except that material may be duplicated by you for your research use or educational purposes in electronic or print form. You must obtain permission for any other use. Electronic or print copies may not be offered, whether for sale or otherwise to anyone who is not an authorised user. 


\title{
Topological state engineering by potential impurities on chiral superconductors
}

\author{
Vardan Kaladzhyan, ${ }^{1,2}$ Joel Röntynen, ${ }^{3}$ Pascal Simon, ${ }^{2}$ and Teemu Ojanen ${ }^{3, *}$ \\ ${ }^{1}$ Institut de Physique Théorique, CEA/Saclay, Orme des Merisiers, 91190 Gif-sur-Yvette Cedex, France \\ ${ }^{2}$ Laboratoire de Physique des Solides, CNRS, Univ. Paris-Sud, Université Paris-Saclay, 91405 Orsay Cedex, France \\ ${ }^{3}$ Department of Applied Physics (LTL), Aalto University, P.O. Box 15100, FI-00076 AALTO, Finland
}

(Received 28 April 2016; published 11 August 2016)

\begin{abstract}
In this work we consider the influence of potential impurities deposited on top of two-dimensional chiral superconductors. As discovered recently, magnetic impurity lattices on an $s$-wave superconductor may give rise to a rich topological phase diagram. We show that a similar mechanism takes place in chiral superconductors decorated by nonmagnetic impurities, thus avoiding the delicate issue of magnetic ordering of adatoms. We illustrate the method by presenting the theory of potential impurity lattices embedded on chiral $p$-wave superconductors. While a prerequisite for the topological state engineering is a chiral superconductor, the proposed procedure results in vistas of nontrivial descendant phases with different Chern numbers.
\end{abstract}

DOI: 10.1103/PhysRevB.94.060505

Introduction. Engineering novel quantum phases of matter with exotic properties is a rapidly growing trend in contemporary physics. The main goal is to employ simpler and wellunderstood ingredients and methods to create more complex structures with desirable properties. Recent promising efforts to realize [1-3] topological superconductivity in nanowire systems $[4,5]$ demonstrate the power of the approach. While it seems unlikely that Nature directly provides us with Majorana quasiparticles that could be employed in quantum information applications [6], it is increasingly probable that those can be achieved in the laboratory. In the spirit of engineering novel controllable states of matter, we show how to realize a complex hierarchy of topological phases with potential impurity superstructures adsorbed on chiral superconductors.

Magnetic atoms on $s$-wave superconductors give rise to Yu-Shiba-Rusinov subgap states [7-10] which have been probed experimentally by scanning tunneling microscopy (STM) [11-14]. Superstructures fabricated from magnetic atoms are currently under active experimental [15-17] and theoretical research [18-33]. Intriguing properties of these systems include the possibility for various one-dimensional (1D) topological superconducting phases with Majorana bound states and rich two-dimensional (2D) topological phases [34-37]. A topologically nontrivial phase is known to arise in 1D ferromagnetic arrays when the underlying superconductor has a strong Rasha spin-orbit coupling or in arrays with helical magnetic textures. In 1D structures there are theoretical arguments why magnetic self-tuning could result in a nontrivial ground state [20-22,28,32,38], though in real systems there are a number of complications. In particular, in 2D structures the nature and tunability of magnetic textures is a delicate and largely unsolved question.

Very recently it was proposed that potential impurities could be utilized to realize interesting topological states in 1D structures [39] and 2D toy models [40]. The procedure requires a non- $s$-wave superconductor host material with chiral or helical pairing components but circumvents the need for specific magnetic textures of adatoms. In the present

*Corresponding author: teemuo@boojum.hut.fi work we provide a microscopic theory of potential impurity structures on chiral superconductors. We show that given a nontrivial chiral superconductor, the potential impurities give rise to a complex hierarchy of distinct nontrivial phases. The Chern number of the phase can be structurally designed by employing different impurities and varying the impurity lattice constant. We illustrate the procedure with a chiral $p$-wave superconductor. However, our results are not restricted to chiral $p$-wave systems and also apply to time-reversal breaking $s+p$ mixtures, higher chiral superconductors, and the artificial $p$-wave model realized in sandwich structures of a 2D semiconductor proximity coupled to an $s$-wave superconductor and a ferromagnetic insulator.

Chiral p-wave systems. Here we formulate the theory describing the system in Fig. 1(a). The bulk electrons in a $2 \mathrm{D}$ spinless $p_{x}+i p_{y}$ superconductor are described by a Bogoliubov-de Gennes (BdG) Hamiltonian

$$
\mathcal{H}_{\mathbf{p}}^{(\text {bulk })}=\xi_{\mathbf{p}} \tau_{z}+\varkappa\left(p_{x} \tau_{x}-p_{y} \tau_{y}\right),
$$

expressed in the Nambu basis $\left(\hat{\Psi}_{p}, \hat{\Psi}_{-p}^{\dagger}\right)$. Here the singleparticle energy is $\xi_{\mathbf{p}}=\frac{p^{2}}{2 m}-\varepsilon_{F}$ with the Fermi energy $\varepsilon_{F}$, and $\varkappa$ is the superconducting $p$-wave pairing amplitude which is taken as real and positive. The Pauli matrices $\tau_{i}$ operate in the particle-hole space. The collection of adatoms act as local potentials described by

$$
\mathcal{H}^{(\mathrm{imp})}(\mathbf{r})=U \tau_{z} \sum_{n} \delta\left(\mathbf{r}-\mathbf{r}_{n}\right),
$$

where $\mathbf{r}_{n}$ are the positions of the atoms and $U$ is the impurity strength. Our treatment is also valid if we consider impurities of a finite size (see the Supplemental Material (SM) [41] for details). The total Hamiltonian consists of the sum $\mathcal{H}=$ $\mathcal{H}^{(\text {bulk })}+\mathcal{H}^{(\mathrm{imp})}$.

Each potential impurity atom binds a single physical subgap state [42-44], which in the BdG formalism is represented by a pair of states at energies $\varepsilon= \pm \frac{\gamma \beta^{2}-\sqrt{1+\beta^{2}\left(1-\gamma^{2}\right)}}{1+\beta^{2}} \Delta_{t}$ for repulsive potential $\beta>0$. For attractive potential $\beta<0$ the solutions are otherwise the same with the exception of a minus sign in front of the square root [41]. Here we have defined quantities $\beta=\pi \nu U, \gamma=\frac{\tilde{\varkappa}}{\sqrt{1+\tilde{\varkappa}^{2}}}, \tilde{\varkappa}=\frac{\varkappa}{v_{F}}$, and $\Delta_{t}=\frac{\varkappa k_{F}}{\sqrt{1+\tilde{\varkappa}^{2}}}$, 
(a)

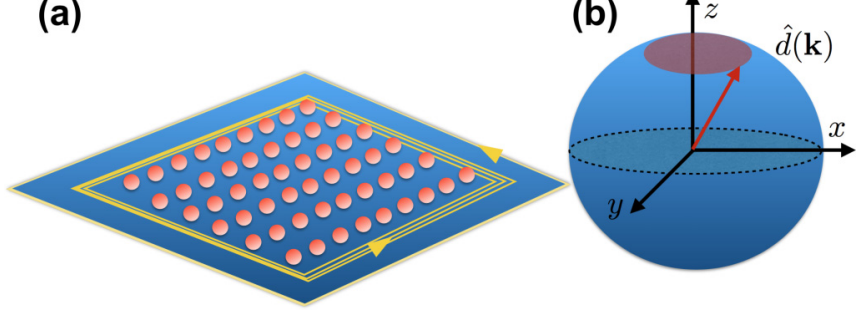

FIG. 1. (a) Schematic representation of the studied system, consisting of potential scatterers deposited on top of a chiral superconductor. The topological phase on the impurity lattice can be be widely modified from that of the underlying compound. (b) In two-band models the Chern number can be illustrated through the motion of the $\hat{d}(\mathbf{k})$ vector on the unit sphere. A long-range hopping translates to high Chern numbers through rapid rotation of $\hat{d}(\mathbf{k})$.

where $v_{F}$ is the Fermi velocity and $v$ the density of states in the bulk [45].

The parameter $\Delta_{t}$ represents the $p$-wave bulk gap determining the coherence length $\xi^{-1}=\frac{\Delta_{t}}{v_{F}}$ and $\beta$ is a dimensionless impurity strength. Strong impurities with $\beta \gg 1$ give rise to deep-lying subgap states close to the Fermi level, while weak impurity states reside near the gap edge. Analogous to the Shiba states in a 2D system [14], the potential impurity wave functions have asymptotic form $e^{i k_{F} r-r / \xi_{E}} / \sqrt{k_{F} r}$ away from the impurity where the decay length is given by $\xi_{E}=$ $\xi / \sqrt{1-\left(E / \Delta_{t}\right)^{2}}$.

When impurity atoms are arranged into a regular array with a lattice constant $a<\xi$, the impurity states bound to a particular atom are hybridized with several nearest neighbors. This leads to the formation of subgap energy bands which support rich topological properties. To study the topological properties of the subgap bands, we formulate effective low-energy theory valid in the deep-dilute impurity regime $\beta \gg 1, \sqrt{k_{F} a} \gg 1$ in the vicinity of the Fermi level. However, as we discussed below, the effective theory yields an exact topological phase diagram which is valid also outside the deep-dilute regime. As outlined in the Supplemental Material [41], a similar procedure that was applied in the Shiba systems $[23,24,29,34]$ results in a description of the impurity lattice in terms of the tight-binding Hamiltonian

$$
H_{m n}=\left(\begin{array}{cc}
h_{m n} & \Delta_{m n} \\
\left(\Delta_{m n}\right)^{\dagger} & -h_{m n}^{*}
\end{array}\right) .
$$

The effective Hamiltonian has an $N \times N$ BdG block structure, where $N$ is the number of impurity atoms. The BdG blocks are given by

$$
\begin{aligned}
h_{m n} & = \begin{cases}\varepsilon_{0}, & m=n \\
A\left(r_{m n}\right), & m \neq n,\end{cases} \\
\Delta_{m n} & = \begin{cases}0, & m=n \\
B\left(r_{m n}\right) \frac{x_{m n}+i y_{m n}}{r_{m n}}, & m \neq n,\end{cases}
\end{aligned}
$$

where the on-site term $\varepsilon_{0}=\Delta_{t}\left(\gamma-\beta^{-1}\right)$ arises from the decoupled impurity energy, $r_{m n}=\left|\mathbf{r}_{m}-\mathbf{r}_{n}\right|$ is the distance between two impurity lattice sites, and $x_{m n}=x_{m}-x_{n}, y_{m n}=$ $y_{m}-y_{n}$. The matrix elements depend on the functions

$$
\begin{aligned}
& A(r)=-\frac{2 \Delta_{t}}{\pi} \operatorname{Re}\left\{\eta K_{0}\left[-i \eta k_{F} r\right]\right\}, \\
& B(r)=-i \frac{2 \Delta_{t}}{\pi} \operatorname{Re}\left\{\eta K_{1}\left[-i \eta k_{F} r\right]\right\},
\end{aligned}
$$

where $K_{i}(x)$ stands for the modified Bessel function of the second kind with index $i$ and $\eta=1+i \tilde{\varkappa}$. The block matrices in Eq. (2) define a hopping model where the amplitudes satisfy asymptotic behavior $\Delta_{m n}, h_{m n} \sim \frac{e^{-r_{m n} / 5}}{\sqrt{r_{m n}}}$ at long distances. The model (1) with entries (2) is a lattice discretized chiral superconductor with rich topological properties discussed below.

Topological properties. The topological phase diagram of the effective model (2) is conveniently extracted in momentum space. For any Bravais lattice we can define Fourier transforms

$$
\begin{aligned}
& d_{x}(\mathbf{k})=\operatorname{Re} \sum_{\mathbf{R}} e^{i \mathbf{k} \cdot \mathbf{R}} \Delta_{\mathbf{R}}, \quad d_{y}(\mathbf{k})=-\operatorname{Im} \sum_{\mathbf{R}} e^{i \mathbf{k} \cdot \mathbf{R}} \Delta_{\mathbf{R}}, \\
& d_{z}(\mathbf{k})=\sum_{\mathbf{R}} e^{i \mathbf{k} \cdot \mathbf{R}} h_{\mathbf{R}},
\end{aligned}
$$

where the sum is over all the lattice vectors $\mathbf{R}=\left(x_{m n}, y_{m n}\right)$. The Hamiltonian can then be written in a simple form $H(\mathbf{k})=$ $\mathbf{d}(\mathbf{k}) \cdot \boldsymbol{\sigma}$ with energies $E(\mathbf{k})= \pm|\mathbf{d}(\mathbf{k})|$. The effective Hamiltonian $H(\mathbf{k})$ describes the gapped two-band model satisfying the particle-hole symmetry $\mathcal{C} H(\mathbf{k})^{*} \mathcal{C}^{-1}=-H(-\mathbf{k})$, where $\mathcal{C}=\sigma_{x} \mathcal{K}$ and $\mathcal{K}$ denotes complex conjugation. The studied model belongs to the Altland-Zirnbauer class $D$, admitting a $\mathbb{Z}$-valued classification by Chern numbers [46]. For two-band models the Chern number is found by evaluating the expression

$$
C=\frac{1}{4 \pi} \int_{\mathrm{BZ}} d^{2} k \frac{\mathbf{d}}{|\mathbf{d}|^{3}} \cdot\left(\frac{\partial \mathbf{d}}{\partial k_{1}} \times \frac{\partial \mathbf{d}}{\partial k_{2}}\right),
$$

which yields integers. The integer value of the Chern number can be visualized through construction depicted in Fig. 1(b). The Hamiltonian defines a unit vector $\hat{d}(\mathbf{k})=\mathbf{d}(\mathbf{k}) /|\mathbf{d}(\mathbf{k})|$ which can be depicted as a point on the surface of a unit sphere. Absolute value of the Chern number measures how many times $\hat{d}(\mathbf{k})$ covers the sphere when $\mathbf{k}=\left(k_{x}, k_{y}\right)$ covers the Brillouin zone of the impurity lattice. The long-range hopping gives rise to rapidly rotating components of $\hat{d}$ vector and thus may lead to chiral states with Chern numbers much larger than unity.

As pointed out in the SM [41], the effective description (1), derived under assumptions of a deep and dilute impurity configuration $\beta \gg 1, \sqrt{k_{F} a} \gg 1$, actually acts as a topological Hamiltonian yielding the exact phase diagram which is also valid outside the deep-dilute regime. This happens because at the topological phase transition, accompanied by the energy gap closing, the effective model (1) becomes exact irrespectively of the values of $\beta$ and $k_{F} a$.

In Fig. 2 we have plotted the topological phase diagram and the energy gap diagram for square lattices. It is clearly evident that the system possesses multiple phases which can be tuned by the separation and strength of the impurities. For higher values of the hybridization parameter $k_{F} a$ the hopping is highly oscillatory, thus leading to more rapid alternation of Figs. 3 and 4 . The generic features of the phase diagrams seem to be in line with the Chern mosaic behavior discovered in magnetic lattices $[34,35]$. For robust states the energy gaps are of the 

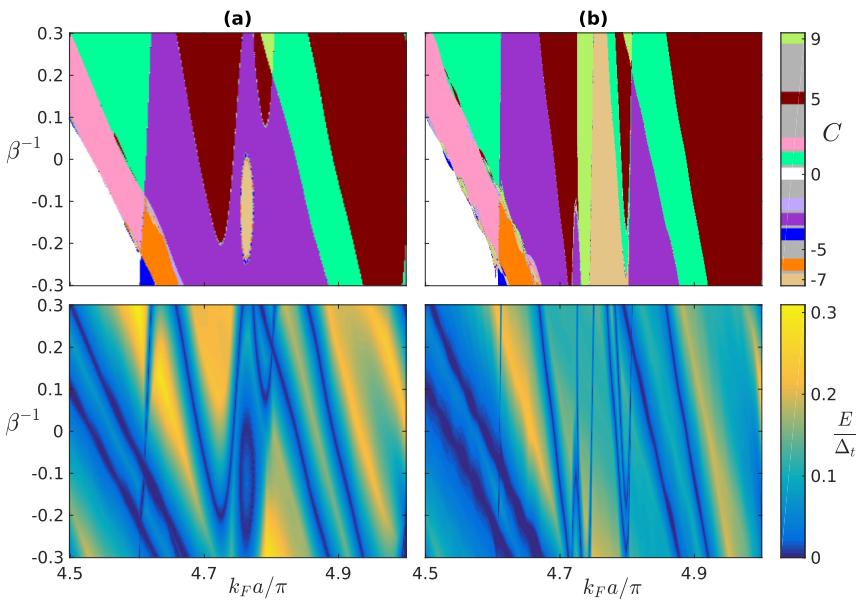

FIG. 2. (a) Chern number (above) and energy gap (below) diagrams for a square lattice of impurities with lattice constant $a$ and coherence length $\xi / a=5$. The quantity $\beta^{-1}$ in the vertical axis controls the strengths of the impurity. The $\beta^{-1}=0$ line, corresponding to infinite impurity potential $|U|=\infty$, divides the repulsive and attractive impurity regions. The horizontal axis $k_{F} a$ controls the hybridization between the bound states centered at different impurity sites. (b) Same as (a) but for coherence length $\xi / a=10$.
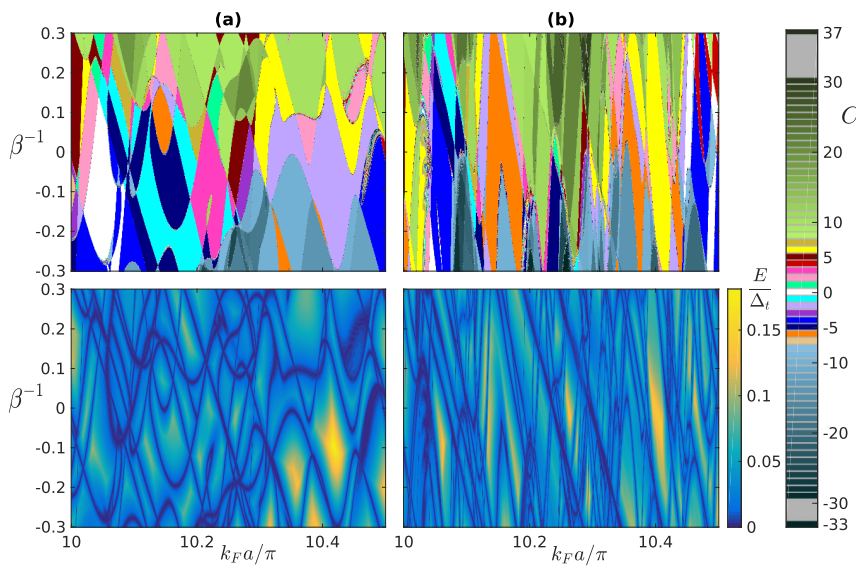

FIG. 3. The same quantities as in Fig. 2 but for larger values of the hybridization parameter $k_{F} a$.
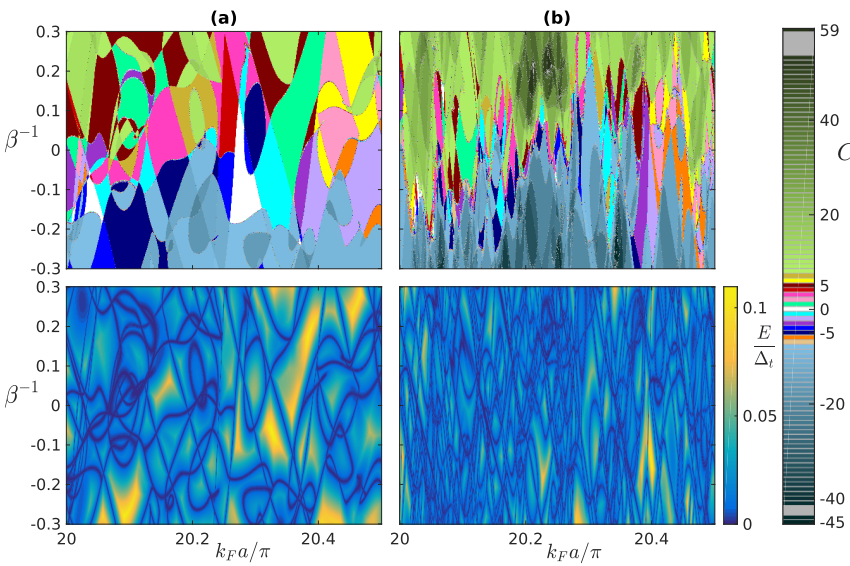

FIG. 4. The same quantities as in Fig. 2 but for larger values of the hybridization parameter $k_{F} a$. order of $0.1-0.2 \Delta_{t}$. Probably larger gaps can be obtained, but studying those would require more elaborate theory as the employed approximations become unreliable. Potential impurity superstructures clearly allow remarkable possibilities for topological state engineering in the studied system without uncertainty associated to the magnetic textures.

We have also diagonalized the system on an infinite strip geometry, where the topological edge modes show up as states traversing the bulk gap. These results are discussed in more detail in the Supplemental Material.

Physical realizations. In the above we have considered potential impurities in spinless chiral $p$-wave superconductors. Our theory can be straightforwardly generalized to the candidate state of $\mathrm{Sr}_{2} \mathrm{RuO}_{4}$ where the opposite spins pair to form $L_{z}=1$ Cooper pairs. Since potential impurities do not mix spin, the $4 \times 4$ model with spin leads to two identical but decoupled $2 \times 2$ blocks of form Eq. (1). The Chern number can be evaluated for each block separately, leading to doubling of the Chern number and the edge modes compared to the spinless case.

However, there are various other candidates for the host materials. The requirements for topological state engineering by potential impurities are rather general and met in a variety of other systems as well. The basic ingredient is that localized potentials must bind subgap bound states in the host material. These bound states in chiral superconductors are generic since Anderson's theorem which guarantees the robustness of $s$-wave superconductors to potential disorder [10] is not operational in time-reversal breaking systems. The second requirement is the phase winding structure $\Delta_{0} e^{i n \varphi_{k}}$, where $\tan \varphi_{k}=k_{y} / k_{x}$, of the gap function of the unperturbed bulk. This will translate to a type of $\left(x_{i j} \pm i y_{i j}\right)^{n} / r_{i j}$ phase structure of the gap function $\Delta_{i j}$ in the effective low-energy BdG Hamiltonian (2), indicating topologically nontrivial superconductivity. In addition, algebraically decaying hopping up to the coherence length is also a universal feature of gapped states. Thus any 2D chiral $(p-, d-, f \ldots$-wave $)$ superconductor satisfies the general requirements and exhibits the characteristic features of the studied chiral $p$-wave model. We note that different crystal structures of the bulk give rise to distinct lattice regulations of chiral gap functions. Also, for a continuum expression $\Delta(\mathbf{k}) \sim\left(k_{x}+i k_{y}\right)^{n}$, corresponding to Chern number $n$, there exists many different lattice versions. However, in the case where the impurity lattice constant is much larger than that of the underlying superconductor, the continuum approximation should prove sufficient.

Dominantly $p$-wave superconductors with $s$-wave pairing amplitude, having a gap structure $\Delta_{s}+\Delta_{p} e^{i \varphi_{k}}$, is also a sufficient starting point for topological state engineering when $\Delta_{p}>\Delta_{s}$. In this case potential-impurity-induced bound states exist [42] and phase winding is inherited to the effective low-energy model. Such $(s+p)$-wave structure is satisfied in the artificial chiral superconductor realized in 2D Rashbacoupled semiconductors sandwiched by an $s$-wave superconductor and a ferromagnetic insulator [47] at sufficiently strong magnetization. Patterning the semiconductor layer with potential impurities or otherwise realizing the potential lattice by applying an external structured potential gate would enable fabrication of nontrivial topological states far beyond Chern number $|C|=1$. 
Chiral and time-reversal breaking superconductors have also been predicted in various other low-dimensional systems. While these have not been observed in experiments so far, it is plausible that some will be realized in the future. At that point a large number of other chiral states will immediately become accessible through topological state engineering by potential superstructures.

Discussion. The bulk topology in a topologically nontrivial state is reflected on its boundary properties. This property could be employed in experimental identification of nontrivial bulk states. The subgap density of states in chiral superconductors arises due to the chiral edge modes as illustrated in Fig. 1(a). Probing the local density of states by STM reveals that the subgap modes are localized on the boundary of the impurity lattice [34]. This method can be employed to show that the impurity lattice is in a different topological phase than the underlying chiral superconductor. Experimental extraction of specific value of the Chern number of a superconductor, while in principle possible, is an unsolved issue at present. However, by fabricating interfaces between lattices of, say, different lattice constants it is possible to compare whether the two adjacent structures belong to the same topological phase. If the structures belong to different phases, there must exist pronounced subgap local density of states at the boundary due to topological edge modes.

The circulating Majorana edge modes, depicted in Fig. 1(a), carry heat in otherwise gapped systems and could find applications in future electronics as chiral heat guides. These waveguides could be designed on top of the superconductor by employing different impurity lattice structures. The Chern number of lattice yields the number of parallel thermal edge channels, so high Chern number states are generally more effective thermal conductors compared to low Chern number states. Also, Majorana bound states trapped in lattice defects could also be interesting from a quantum information point of view. While the applications of chiral superconductors are still emerging, our work points to a conceptually simple method to obtain them in nanofabricated structures.

Conclusions. In this work we proposed a method to engineer topological states by potential impurities deposited on $2 \mathrm{D}$ chiral superconductors. In particular, we presented a microscopic theory of chiral $p$-wave superconductors with impurity lattices. This allowed us to calculate the topological phase diagram for general impurity strengths and hybridization. Our results have remarkable conceptual and practical consequences: given a 2D chiral superconductor, it is possible to fabricate a large number of nontrivial descendant states by a straightforward procedure. Because potential-induced subgap states are generic in time-reversal breaking superconductors and superfluids, our results have universal appeal irrespective of the platform and microscopic details of the chiral state.

Acknowledgments. The authors acknowledge Aalto Science-IT project for the computational resources and the Academy of Finland (T.O.) and the Finnish Cultural Foundation (J.R.) for support. P.S. would like to acknowledge the financial support from the French Agence Nationale de la Recherche through the contract Mistral.
[1] V. Mourik, K. Zuo, S. M. Frolov, S. R. Plissard, E. P. A. M. Bakkers, and L. P. Kouwenhoven, Science 336, 1003 (2012).

[2] A. Das, Y. Ronen, Y. Most, Y. Oreg, M. Heiblum, and H. Shtrikman, Nat. Phys. 8, 887 (2012).

[3] S. M. Albrecht, A. P. Higginbotham, M. Madsen, F. Kuemmeth, T. S. Jespersen, J. Nygard, P. Krogstrup, and C. M. Marcus, Nature (London) 531, 206 (2016).

[4] R. M. Lutchyn, J. D. Sau, and S. Das Sarma, Phys. Rev. Lett. 105, 077001 (2010).

[5] Y. Oreg, G. Refael, and F. von Oppen, Phys. Rev. Lett. 105, 177002 (2010).

[6] C. Nayak, S. H. Simon, A. Stern, M. Freedman, and S. Das Sarma, Rev. Mod. Phys. 80, 1083 (2008).

[7] L. Yu, Acta Phys. Sin. 21, 75 (1965).

[8] H. Shiba, Prog. Theor. Phys. 40, 435 (1968).

[9] A. I. Rusinov, JETP Lett. 9, 85 (1969).

[10] A. V. Balatsky, I. Vekhter, and J.-X. Zhu, Rev. Mod. Phys. 78, 373 (2006).

[11] A. Yazdani, B. A. Jones, C. P. Lutz, M. F. Crommie, and D. M. Eigler, Science 275, 1767 (1997).

[12] S.-H. Ji, T. Zhang, Y. S. Fu, X. Chen, X. C. Ma, J. Li, W. H. Duan, J. F. Jia, and Q. K. Xue, Phys. Rev. Lett. 100, 226801 (2008).

[13] N. Hatter, B. W. Heinrich, M. Ruby, J. I. Pascual, and K. J. Franke, Nat. Commun. 6, 8988 (2015).

[14] G. C. Ménard, S. Guissart, C. Brun, S. Pons, V. S. Stolyarov, F. Debontridder, M. V. Leclerc, E. Janod, L. Cario, D. Roditchev, P. Simon, and T. Cren, Nat. Phys. 11, 1013 (2015).
[15] S. Nadj-Perge, I. K. Drozdov, J. Li, H. Chen, S. Jeon, J. Seo, A. H. MacDonald, B. Andrei Bernevig, and Ali Yazdani, Science 346, 602 (2014).

[16] R. Pawlak, M. Kisiel, J. Klinovaja, T. Meier, S. Kawai, T. Glatzel, D. Loss, and E. Meyer, arXiv:1505.06078.

[17] M. Ruby, F. Pientka, Y. Peng, F. von Oppen, B. W. Heinrich, and K. J. Franke, Phys. Rev. Lett. 115, 197204 (2015).

[18] T. P. Choy, J. M. Edge, A. R. Akhmerov, and C. W. J. Beenakker, Phys. Rev. B 84, 195442 (2011).

[19] S. Nadj-Perge, I. K. Drozdov, B. A. Bernevig, and A. Yazdani, Phys. Rev. B 88, 020407(R) (2013).

[20] B. Braunecker and P. Simon, Phys. Rev. Lett. 111, 147202 (2013).

[21] J. Klinovaja, P. Stano, A. Yazdani, and D. Loss, Phys. Rev. Lett. 111, 186805 (2013).

[22] M. M. Vazifeh and M. Franz, Phys. Rev. Lett. 111, 206802 (2013).

[23] F. Pientka, L. I. Glazman, and F. von Oppen, Phys. Rev. B 88, 155420 (2013).

[24] F. Pientka, L. I. Glazman, and F. von Oppen, Phys. Rev. B 89, 180505 (2014).

[25] K. Pöyhönen, A. Westström, J. Röntynen, and T. Ojanen, Phys Rev. B 89, 115109 (2014).

[26] J. Röntynen and T. Ojanen, Phys. Rev. B 90, 180503 (2014).

[27] A. Heimes, P. Kotetes, and G. Schön, Phys. Rev. B 90, 060507(R) (2014).

[28] I. Reis, D. J. J. Marchand, and M. Franz, Phys. Rev. B 90, 085124 (2014). 
[29] P. M. R. Brydon, S. Das Sarma, H.-Y. Hui, and J. D. Sau, Phys. Rev. B 91, 064505 (2015).

[30] A. Heimes, D. Mendler, and P. Kotetes, New J. Phys. 17, 023051 (2015).

[31] A. Westström, K. Pöyhönen, and T. Ojanen, Phys. Rev. B 91, 064502 (2015).

[32] B. Braunecker and P. Simon, Phys. Rev. B 92, 241410 (2015).

[33] K. Pöyhönen, A. Westström, and T. Ojanen, Phys. Rev. B 93, 014517 (2016).

[34] J. Röntynen and T. Ojanen, Phys. Rev. Lett. 114, 236803 (2015).

[35] J. Röntynen and T. Ojanen, Phys. Rev. B 93, 094521 (2016).

[36] J. Li, T. Neupert, Z .J. Wang, A. H. MacDonald, A. Yazdani, and B. A. Bernevig, Nat. Commun. 7, 12297 (2016).

[37] S. Nakosai, Y. Tanaka, and N. Nagaosa, Phys. Rev. B 88, 180503 (2013).

[38] M. Schecter, K. Flensberg, M. H. Christensen, B. M. Andersen, and J. Paaske, Phys. Rev. B 93, 140503 (2016).
[39] T. Neupert, A. Yazdani, and B. A. Bernevig, Phys. Rev. B 93, 094508 (2016).

[40] L. Kimme and T. Hyart, Phys. Rev. B 93, 035134 (2016).

[41] See Supplemental Material at http://link.aps.org/supplemental/ 10.1103/PhysRevB.94.060505 for technical details of the derivation of the studied model, its generalizations and numerical results.

[42] V. Kaladzhyan, C. Bena, and P. Simon, Phys. Rev. B 93, 214514 (2016).

[43] V. Kaladzhyan, C. Bena, and P. Simon (unpublished).

[44] Y. Kim, J. Zhang, E. Rossi, and R. M. Lutchyn, Phys. Rev. Lett. 114, 236804 (2015).

[45] These results are derived by linearizing the dispersion relation around the Fermi momentum (see [42]).

[46] A. P. Schnyder, S. Ryu, A. Furusaki, and A. W. W. Ludwig, Phys. Rev. B 78, 195125 (2008); S. Ryu, A. P. Schnyder, A. Furusaki, and A. W. W. Ludwig, New J. Phys. 12, 065010 (2010).

[47] J. D. Sau, R. M. Lutchyn, S. Tewari, and S. Das Sarma, Phys. Rev. Lett. 104, 040502 (2010). 